Steuer, Dunja; Bolten, Jens; Grießbach, Gert:

Effiziente Gestaltung komplexer adaptiver Schätzalgorithmen durch objektorientierte Programmierung

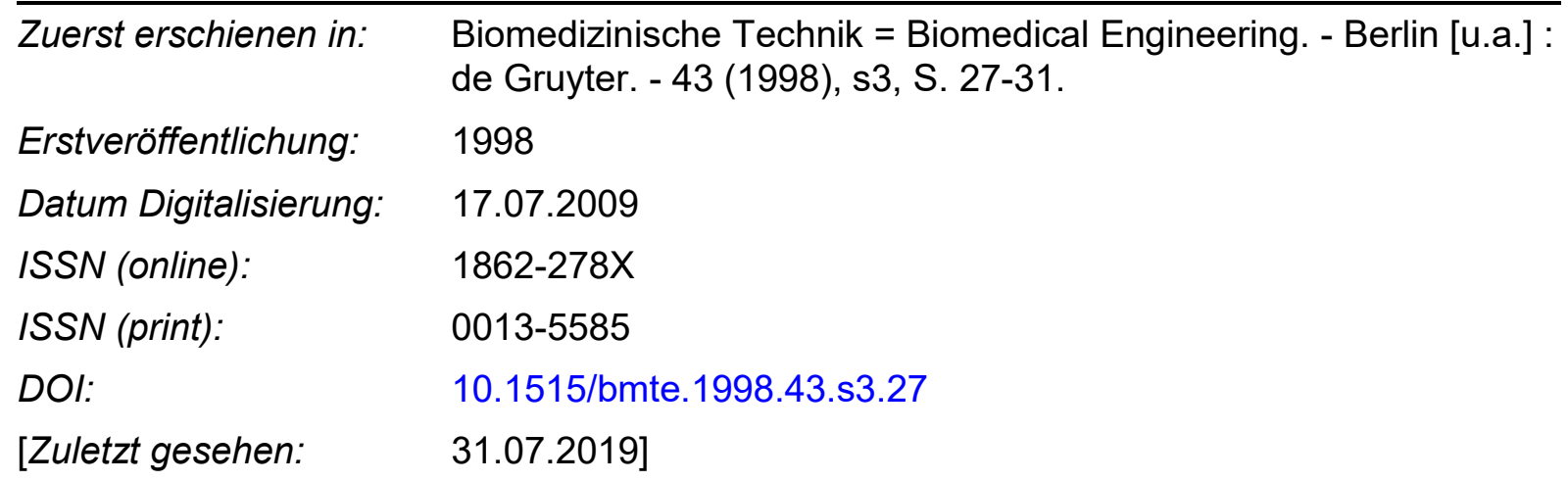

„Im Rahmen der hochschulweiten Open-Access-Strategie für die Zweitveröffentlichung identifiziert durch die Universitätsbibliothek IImenau."

"Within the academic Open Access Strategy identified for deposition by IImenau University Library."

„Dieser Beitrag ist mit Zustimmung des Rechteinhabers aufgrund einer (DFGgeförderten) Allianz- bzw. Nationallizenz frei zugänglich."

"This publication is with permission of the rights owner freely accessible due to an Alliance licence and a national licence (funded by the DFG, German Research Foundation) respectively."

\section{DFG}

Nationallizenzen 


\title{
Effiziente Gestaltung komplexer adaptiver Schätzalgorithmen durch objektorientierte Programmierung
}

\author{
Steuer D. Bolten J. Grießbach G. \\ Technische Universität Ilmenau, Institut für Biomedizinische Technik und Informatik, D-98684 Ilmenau
}

\section{EINLEITUNG}

Mit der stetig zunehmenden Leistungsfähigkeit moderner Rechentechnik steigen auch die Anforderungen an Analyseverfahren für komplexere Signal- und Bildverarbeitungsprobleme. Durch die Nutzung moderner Informationsverarbeitungsverfahren, zu denen zum Beispiel die objektorientierten Technologien gehören, erhofft man sich wesentliche neue Impulse. Der vorliegenden Beitrag greift diese Idee auf und zeigt sowohl neue Ansätze zur Gestaltung von Analyseverfahren als auch neuartige Analyseverfahren selbst. Zur Anwendung kommen dazu die bereits aus vielen Problemstellungen der Biosignalverarbeitung bekannten und bewährten adaptiven rekursiven Schätzverfahren, die sich durch ihre Struktur für eine Realisierung in objektorientierter Programmierung anbieten. Veranschaulicht wird die Vorgehensweise an Beispielen aus der Kognitionsforschung, die im Rahmen eines DFG-Projektes untersucht wurden.

\section{METHODE \\ Adaptive rekursive Schätzverfahren}

Wesentlicher Arbeitsfeld der Biosignalanalyse ist die Untersuchung stochastischer Signale, die oftmals durch das Vorhandensein von Instationaritäten gekennzeichnet sind. Da diese Instationaritäten aber häufig von diagnostischem Interesse sind, erinnert sei hier z.B. an Spike-WaveKomplexe in der Epilepsie-Untersuchung, müssen Signalverarbeitungsstrategien zur Anwendung kommen, die diese Informationen berücksichtigen. Die konventionelle Spektralanalyse hat unter den genannten Voraussetzungen ihre Grenzen. Moderne Analyseverfahren können diese Probleme umgehen. Der Ideenapparat der Stochastischen Approximation kann zur Konstruktion adaptiver rekursiver Schätzverfahren genutzt werden. Ausgehend von stationären ergodischen Prozessen werden Parameter geschätzt. Bei Abweichung der Schätzung vom bekannten Zustand können Instationaritäten detektiert und klassifiziert werden. Adaptive rekursive Schätzverfahren lassen sich allgemein in nachfolgender Gestalt konstruieren:

$$
\begin{aligned}
& S_{0}=s_{0} \quad \text { (Startwert) } \\
& S_{n+1}=S_{n}+c_{n} \cdot K\left(S_{n}, x_{n+1}\right) .
\end{aligned}
$$

Dabei bezeichnet $X=\left\{x_{i}\right\}_{i=0,1,2, \ldots}$ die Zeitreihe des stochastischen Signals, $K$ stellt einen Korrekturterm dar und $\left\{c_{n}\right\}_{n=0,1,2, \ldots}$ ist eine Zahlenfolge, die durch bestimmte Bedingungen (siehe [1]) zur Konvergenz führt. Verzichtet man durch Ersetzen der Folge $\{c\}$ durch eine Konstante auf die Konvergenz des Schätzverfahrens, ist man in der Lage, sehr schnell auf Signalveränderungen zu reagieren. Dabei hat eine große Konstante $c$ eine schnellere Anpassung zur Folge als eine kleine Konstante, wobei sich in diesem Fall aber die Streuung der Schätzfunktion reduziert. Einige einfache adaptive Schätzungen sind z.B. der Mittelwert, ein 2.statistisches Moment oder der Quantilwert.

Adaptiver Mittelwert

$$
\begin{aligned}
& M_{0}=m_{0} \quad \text { (Startwert) } \\
& M_{n+1}=M_{n}-c \cdot\left(M_{n}-x_{n+1}\right) .
\end{aligned}
$$

Adaptives 2.Statistisches Moment

$$
\begin{aligned}
& E_{0}=e_{0} \quad \text { (Startwert) } \\
& E_{n+1}=E_{n}-c \cdot\left(E_{n}-x_{n+1}^{2}\right) .
\end{aligned}
$$

Adaptiver Quantilwert

$$
\begin{aligned}
& Q_{0}=q_{0} \quad \text { (Startwert) } \\
& Q_{n+1}= \begin{cases}Q_{n}+c^{*} \alpha & \text { wenn } x_{n+1} \geq Q_{n} \\
Q_{n}+c *(1-\alpha) & \text { wenn } x_{n+1}<Q_{n}\end{cases}
\end{aligned}
$$

Rechentechnisch können diese Verfahren aufgrund ihrer Struktur sehr leicht implementiert werden. Die objektorientierten Technologien stellen dazu ein effektives Werkzeug dar. Unter Anwendung dieser Techniken ist eine Konstruktion komplexerer adaptiver Schätzungen, z.B. in Form von Vektoren oder Matrizen, aus den aufgeführten einfachen Schätzungen zu konstruieren.

\section{Objektorientierte Programmierung}

Die vorgestellten adaptiven Verfahren lassen sich mit Hilfe objektorientierter Programmierung sehr effizient gestalten und weiterentwickeln, um neue Schätzgrößen zu gewinnen. Eine mögliche Realisierung einer abstrakten Klasse "TAdapt" stellt Abb.1 dar. Das Verhalten einer gewünschten adaptiven Schätzfunktion wird in abgeleiteten Klassen von TAdapt durch Überschreiben der abstrakten Methode "correct" erreicht. Wie bereits 


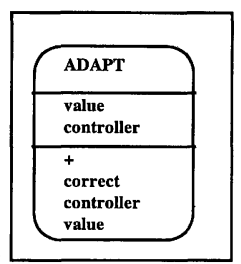

Abb.1: Abstrakte Klasse TAdapt

angedeutet, hat die Wahl der Adaptionskonstanten entscheidenden Einfluß auf die Charakteristik des Schätzalgorithmus. Die Steuerung kann z.B. durch konvergente, beschleunigte, fuzzy-gesteuerte oder NNbasierende Algorithmen erfolgen (NN-Neuronales Netz). In objektorientierter Technologie wird dem durch die Einführung einer abstrakten Klasse "TController" Rechnung getragen, durch die verschiedenste Adaptionsalgorithmen abgeleitet werden können (Abb.2). So besitzt jedes abgeleitete adaptive Objekt eine eigene Steuerung der Adaptionskonstanten.

Aufbauend auf diese Vorbetrachtungen lassen sich einfache Schätzalgorithmen konstruieren. Dazu gehören der adaptive Mittelwert, ein zweites Moment oder der Quantilwert (siehe vorhergehenden Abschnitt).
Viele der bereits erprobten adaptiven Schätzalgorithmen zeichnen sich aber durch eine komplexere Struktur aus. Wünschenswert ist es, auch diese Kenngrößen objektorientiert zu gestalten. Dazu bieten sich grundsätzlich 3 Möglichkeiten an. Die einfachste Form ist die arithmetische Verknüpfung einfacher adaptiver Objekte, wie z.B. bei der adaptiven Varianz (siehe Gl.5).

\section{Adaptive Varianz}

$$
V_{\left[c_{1}, c_{2}\right]}(X)=E^{c_{2}}(X)-\left(M^{c_{1}}(X)\right)^{2} .
$$

Eine weitere Form der Verknüpfung ist die Zusammenfassung adaptiver Objekte in Vektoren. Das wiederum führt zur 3.Form der Verknüpfung, der Zusammenfassung in Matrizen. Diese Form eignet sich besonders für Aufgabenstellungen der Bildverarbeitung, da ein Bild ebenfalls als Matrix aufgefaßt werden kann.

Am Beispiel der adaptiven Varianz soll die Vorgehensweise bei der arithmetischen Aggregation näher erläutert werden. Abb.3 zeigt die abstrakte Darstellung. Die so geschaffene Klasse hält einen Arithmetikmanager und eine Liste adaptiver Objekte als Instanzvariablen. Diese Liste wird bei der Instanziierung durch konkrete Objekte ersetzt und durch Senden der arithmetischen Botschaft die Art der Verknüpfung der Listenelemente festgelegt. Anschließend kann durch

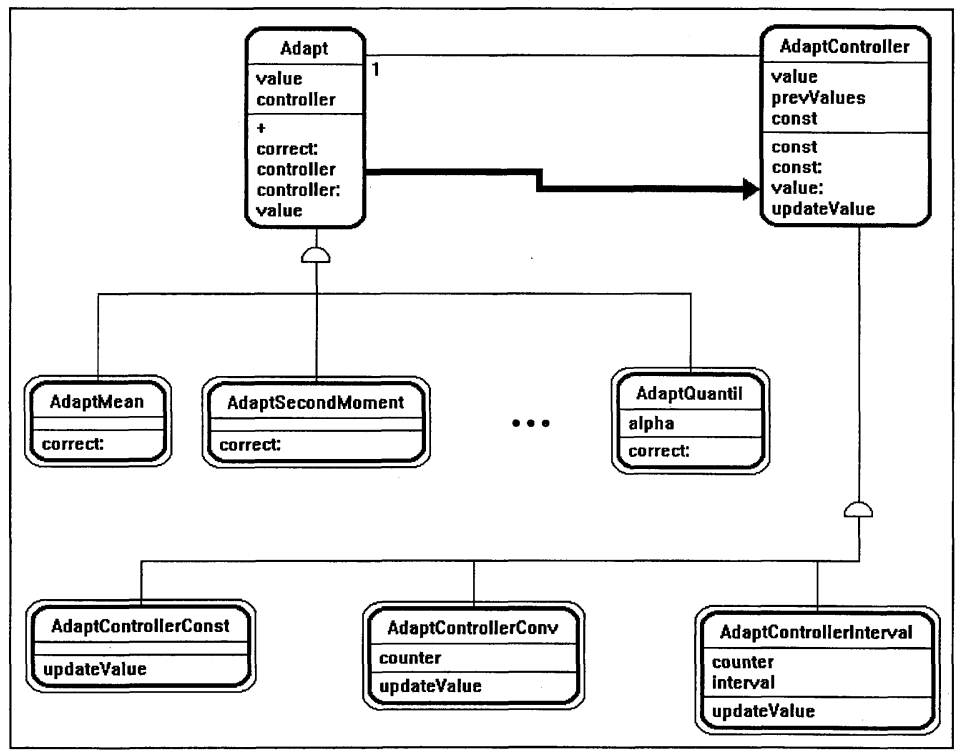

Abb.2: Allgemeine Struktur zur Objektverknüpfung 
Senden der "+"-Botschaft bei anliegendem Meßwert "X" ein neuer Wert der Schätzgröße ermittelt. Dabei schätzt jedes Einzelobjekt der Liste seinen neuen Schätzwert,

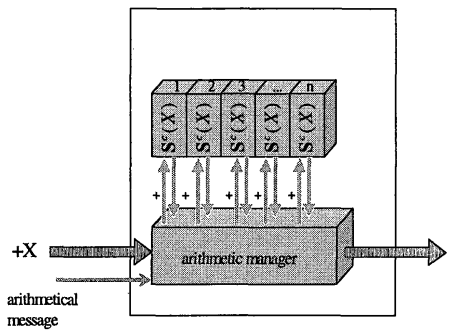

Abb.3: Aggregation adaptiver Objekte durch arithmetische Verknüpfung

wobei die Einzelergebnisse durch den Arithmetikmanager verarbeitet und ausgegeben werden. Abb.4 demonstriert diese Vorgehen konkret für die adaptive Varianz.

Während der Instanziierung wird die Objektliste mit einem Objekt des 2.Statistischen Moments und einem MittelwertObjekt gefüllt. Die arithmetische Botschaft "1-2*2" bedeutet, daß vom Schätzwert des 1.Objekts das Quadrat

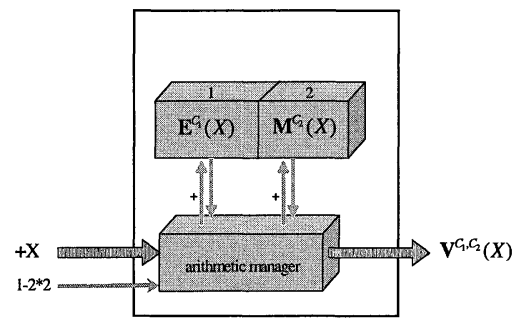

Abb.4: Berechnung der adaptiven Varianz aus einfachen adaptiven Objekten

des Schätzwerts des 2.Objekts abgezogen wird. Die 't'Botschaft bewirkt mit dem aktuellen Meßwert " $\mathrm{X}$ " die Berechnung der Varianz auf der Basis der Einzelschätzungen. Anzumerken ist noch, daß jedes Einzelobjekt eine eigene Steuerung der Adaptionskonstanten besitzt.

Ausgehend von diesen Überlegungen lassen sich jetzt eine Vielzahl komplexerer Schätzungen konstruieren. Der Vorteil dieser Methodik liegt in der anwenderspezifischen Entwicklung neuartiger Analyseverfahren bzw. in einer effektiven Gestaltung bekannter Schätzungen.

\section{ERGEBNISSE}

Die bereits angedeuteten Möglichkeiten der Verknüpfung adaptiver Objekte in Vektor- oder Matrixform eröffnen neue Vorgehensweisen in der Signalverarbeitung. So lassen sich mehrkanalige Signale durch einen Vektor von Mittelwertschätzungen effektiv z.B. tiefpaßfiltern. Dabei kann jedes einzelne Vektorelement individuell in Bezug auf das Adaptionsverhalten gesteuert werden, da jeder Mittelwert z.B. mit einem anderen adaptiven Controller ausgelegt werden kann. Damit kann z.B. auf unterschiedliche Signalcharakteristika eingegangen werden. Die Konstruktion eines Vektors von adaptiven Mittelwertobjekten kann aber auch zur Konstruktion von neuartigen Parameterschätzungen benutzt werden. So läßt sich z.B. eine adaptive Schätzung eines Histogramms wie folgt realisieren:

$$
H_{X}=\left[\mathbf{M}^{c}\left(I_{K_{1}}(X)\right) \ldots, \mathbf{M}^{c}\left(I_{K_{k}}(X)\right)\right]
$$

wobei $I_{K}(X)=\left\{I_{K}\left(x_{i}\right)\right\}_{i=0,1,2, \ldots}$ die Indikatorfunktion zur Ermittlung der Klassenzugehörigkeit des jeweiligen Meßwertes darstellt. In [2] wird diese Schätzung zur Detektion von Veränderungen in Verteilungseigenschaften von Signalen benutzt.

Eine Anwendung in der Bildverarbeitung zur Analyse von Bildfolgen ist ebenfalls denkbar, da das Histogramm ein wesentlicher Bildbeschreibungsparameter ist. Der Unterschied zur vorherigen Applikation besteht nur in der Anzahl der Eingangskanäle zur Histogrammermittlung. Ein Anwendungsfeld für die Bildverarbeitung wird derzeit in einem DFG-Projekz zur Mikroanalyse von Denkprozessen untersucht [4]. Dabei wird während kognitiver Anforderungen ein mehrkanaliges EEG abgeleitet und dieses bzw. daraus berechnete Parameter in EEG-Parameter-Mapsequenzen visualisiert. Diese Sequenzen sollen segmentiert werden, um Aktivitätsphasen während des Denkprozesses zu detektieren.

Eine weitere Möglichkeit ergibt sich für die Verbundanalyse zweier Signale. Die adaptive Schätzung der Kovarianz zweier stochastischer Null-Mittel-Signale läßt sich folgendermaßen darstellen:

$$
\mathbf{K}^{c}(X, Y)=\left[\mathbf{M}^{c}\left(X \otimes \mathbf{L}_{0}(Y)\right), \ldots, \mathbf{M}^{c}\left(X \otimes \mathbf{L}_{n}(Y)\right)\right]
$$

wobei

$\mathbf{L}_{\tau}(Y): \quad L_{\tau}\left(\left\{y_{i}\right\}_{i=0,1,2, \ldots, N-1}\right)=\left\{y_{i+\tau}\right\}_{i=0,1,2, \ldots,}$ einen

Verschiebungsoperator über $\mathrm{Y}, \otimes$ einen Operator für eine elementweise Multiplikation der Signalfolge und $\mathbf{M}^{c}$ wieder einen adaptiven Mittelwertoperator bezeichnet. In [3] wird untersucht, welche neuen Möglichkeiten sich durch die Berechnung der Korrelation auf adaptivem Weg für die Biosignalanalyse ergeben. Da die Korrelation auch als Grundlage zur Berechnung der 
spektralen Kenngröße Kohärenz angesehen werden kann, wird gegenwärtig im genannaten DFG-Projekt ebenfalls untersucht, wie eine adaptive Schätzung der Kohärenz effizient gestaltet werden kann. Der Vorteil gegenüber einer bereits bestehenden Berechnungsmöglichkeit über eine adaptive ARMA-Modellierung [5] könnte in einer wesentlich schnelleren Berechnung der Kohärenz liegen.

Wie bereits erwähnt, lassen sich auch Matrizen adaptiver Schätzungen konstruieren. Die Anwendung in der Bildverarbeitung liegt hier nahe. Abb.5 zeigt eine

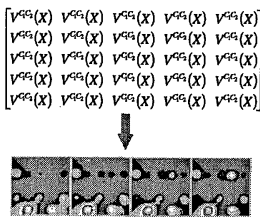

Map-Sequenz der adaptiven Varianz

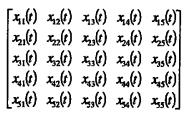

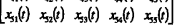
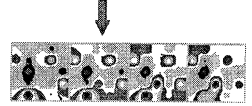

Sequenz der Ausgangsmaps
Abb.5: Berechnung der adaptiven Varianz einer Bildmatrix

Anwendung, um Veränderungen in fortlaufenden Bildmatrizen zu detektieren. Als Ähnlichkeitsmaß kann die Varianz benutzt werden. Dazu wird in der Größe der Bildmatrizen eine Matrix adaptiver Varianzschätzungen initialisiert. Jedes Pixel wird jetzt in seinem zeitlichen Verlauf über die Bildfolge der Varianzschätzung zugeführt. Ändert sich seine Farbe nicht oder kaum, führt das zu einer niedrigen Varianz. Visualisiert man sich den Varianzverlauf wieder, so können große Flächen mit niedrigen Farbwerten als Gebiete mit geringer Änderung detektiert werden. Zur Anwendung kommen diese Untersuchungen wieder zur Segmentierung von Mapsequenzen. Auch hier kann wieder jedes einzelne Matrixelement eine eigene Controllerstruktur besitzen, wodurch eine unterschiedliche Bewertung von einzelnen Gebieten (z.B. Rändern) möglich ist.

Unter Anwendung moderner Informationsverarbeitungstechnologien muß es jetzt auch möglich sein, die auf gleichartigen Grundverfahren beruhenden unterschiedlichen Applikationen durch ein und dieselbe Software $\mathrm{zu}$ realisieren und gleichzeitig so flexibel $\mathrm{zu}$ sein, daß bisher nicht betrachtete Anwendungen mit diesem System ebenfalls analysiert werden können. Abb.6 stellt diese Forderung noch einmal dar. Unter Nutzung objektorientierter Verfahren kann diese Forderung erfüllt werden. Dem Anwender wird eine Software zur Verfügung gestellt, die nur abstrakte Funktionalität enthält. Das bedeutet, daß einfache adaptive Schätzungen, wie Mittelwert, 2.statistisches Moment usw., sowie abstrakte Strukturen von TVektor und TMatrix zur Aufnahme adaptiver Objekte als Klassendefinitionen zur Verfügung gestellt werden. Der Programmierer weiß während der Programmentwicklung nicht, mit welchen Controllern z.B. Mittelwertschätzungen ausgeführt werden sollen, oder welche Schätzungen in eine Vektor-

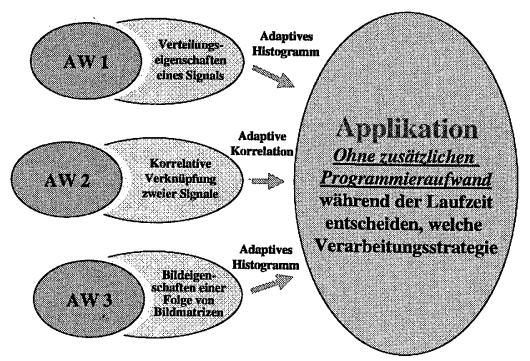

Abb.6: Anforderungen an eine flexible Signalverarbeitungssoftware

oder Matrixstruktur aufgenommen werden sollen. Der Anwender entscheidet erst zur Laufzeit über den Inhalt seiner Signalverarbeitung. Abb.7 demonstriert diese Vorgehensweise schematisch.

Durch Einfügen eines TVektor-Elementes in die Verabeitungskette wird eine Instanz der Klasse TVektor

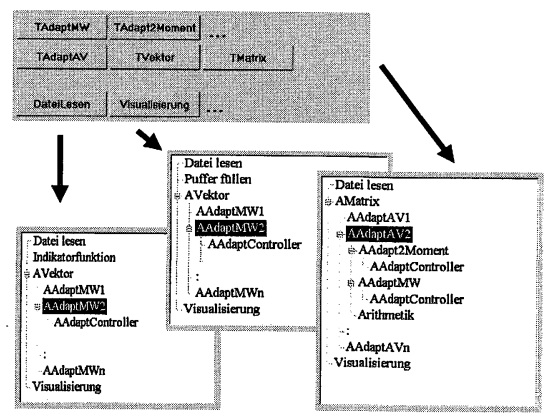

Abb.7: Instanziierung abstrakter adaptiver Objekte während der Laufzeit

gebildet. Dabei ist noch nicht bekannt, welche Objekte dieser Vektor jetzt aufnimmt. Durch Einfügen von Mittelwertobjekten werden diese ebenfalls instanziiert und können zusätzlich noch mit verschiedenen Controllern zur Steuerung des Adaptionsverhaltens ausgestattet werden.

Durch diese interaktive Zusammenstellung der Verarbeitungselemente können sehr schnell neue Strategien ausprobiert werden, ohne daß erst ein neues Programm entwickelt werden muß. Für den Anwender ergibt sich demzufolge ein effizientes Werkzeug in der Signalanalyse.

\section{DISKUSSION}

Die vorgestellte Methodik gestattet es, auch in der Biosignalanalyse moderne Informationsverarbeitungstechnologien, wie sie die objektorientierten Verfahren 
darstellen, effektiv anzuwenden. Durch interaktive Gestaltung der Signalverarbeitungsstrategien wird erst zur Laufzeit der Signalverarbeitungssoftware festgelegt, welche adaptiven Objekte zu welchen komplexen Schätzungen zusammengesetzt werden. Sowohl für den Programmierer als auch für den Anwender ist kein oder nur minimaler zusätzlicher Programmieraufwand nötig, um neue Verfahren zu entwickeln, da bestehende Software wiederverwendet werden und durch Vererbung weiterentwickelt werden kann. Das führt zur Senkung von Kosten in der Entwicklung von Signalverarbeitungssoftware. Desweiteren kann eine hohe Qualität der Software erreicht werden, da man mit bereits getesteten und somit möglichst fehlerfreien Strukturen arbeitet.

\section{LITERATUR}

[1] Grießbach G. (1990): Computerorientierte Meßstochastik in der Technischen Diagnose und dem Signalmapping. Habilitation, FSU Jena

[2] Möller, E., Grießbach, G., Peters, C., Witte, H.(1997) :Adaptive Verfahren zur Beschreibung und zum Vergleich von Verteilungen biologischer Signale. Biomedizinische Technik, Band 42, Ergänzungsband 2, S. 25-26, ISSN 0939-4990

[3] Grießbach, G., Grießbach, U. (1997): Adaptive Korrelationsverfahren in der Biosignalanalyse. In: Muche, R., Büchele, G., Harder, D., Gaus, W. (Hrsg.), Medizinische Informatik, Biometrie und Epidemiologie (82), MMV Medizin Verlag München, (42. Jahrestagung der GMDS in Ulm), ISBN 3-8208-1315-2

[4] Steuer D., Grießbach G. (1997): Komplexe adaptive Schätzalgorithmen in der Biosignalverarbeitung. Biomedizinische Technik, Band 42, Ergänzungsband 2, S.21-22, ISSN 0939-4990

[5] Schack, B. and Krause, W. (1995): Dynamic Power and Coherence Analysis of Ultra ShortTerm Cognitive Processes - A Methodical Study. Brain Topography 8, 127-136

Gefördert durch das DFG-Projekt "Mikroanalyse von Denkprozessen", Nr. GR1555/1-2. 